ermittelt. Es lassen sich so in einem einzigen Pt-Tiegel 2500-3000 Schmelzaufschlüsse durchführen; die Kosteneinsparung ist dementsprechend erheblich.

Dr. Knut Ohls

Hoesch Hüttenwerke $A G$

D-4600 Dortmund

Postfach 902

Deutschland

\section{Quantitative Bestimmung organischer Substanzen mit Hilfe der Massenspektrometrie}

Quantitative Determination of Organic Substances by Means of Mass Spectrometry

\section{W. D. KOLLER}

Institut für Chemie und Technologie der Bundesforschungsanstalt für Lebensmittelfrischhaltung Karlsruhe

Eingegangen am 22. Oktober 1971

Bringt man eine feste Substanz über das Direkteinlaßsystem in die Ionenquelle eines Massenspektrometers, so verdampft sie bei genügend hoher Temperatur und liefert das für sie charakteristische reproduzierbare Massenspektrogramm. Die einzelnen Bruchstücke stehen dabei sowohl untereinander als auch zur eingebrachten Substanzmenge in einem weitgehend konstanten Verhältnis. Das bedeutet, daß z. B. eine Verdoppelung der Substanzmenge auch eine Verdoppelung der Fläche des massenspektrometrischen Peaks zur Folge hat. Auf dieser Beziehung kann eine einfache quantitative Bestimmungsmethode aufgebaut werden. Man geht dabei so vor, daß man nicht das ganze Massenspektrum aufnimmt, sondern stellt das Massenspektrometer so ein, daß nur ein Bruchstück (von möglichst hoher Intensität, wegen der Nachweisempfindlichkeit) mit der Masse ,,$X^{\prime *}$ erfaßt wird. In dem Umfang, in dem die Substanz verdampft und unter dem Ionenbeschuß aufgespalten wird, entsteht auch dieses Bruchstück, dessen quantitative Zunahme bzw. spätere Abnahme sich mittels eines Pult-Schreibers verfolgen und aufzeichnen läßt; das Kurvenbild, das man dabei erhält, entspricht etwa dem einer gas-chromatographischen Kurve. Für die Aufstellung von Eichkurven müssen die Peaks, da sie unsymmetrisch sind, planimetriert und die Flächen (in Quadratzentimeter) dann gegen die Substanzmenge (in Nanogramm) aufgetragen werden.
Das Verfahren wurde mit Melissinsäure $\left(\mathrm{C}_{30: 0}\right)$ als Modellsubstanz überprüft. Mit Hilfe einer Eichlösung (10 mg Melissinsäure in $50 \mathrm{ml}$ Tetrahydrofuran) wurden zwischen 400 und $1600 \mathrm{ng}$ ins Massenspektrometer gebracht. Da kein Massenmarkierer vorhanden war, konnte der Wert für die als Bestimmungsgröße ausgewählte Masse ,, $X^{\prime \prime}(X=m / e$ $\pm \Delta m(e)^{1}$ von einer Versuchsreihe zur anderen nicht exakt reproduziert werden; während einer einzelnen Versuchsreihe blieb die Einstellung für , $\mathrm{X}^{\prime}$ jedoch konstant. Bei einer quantitativen Bestimmung muß deshalb stets eine Eichkurve mitaufgenommen werden.

Die bei diesen Versuchsreihen erhaltenen Eichkurven waren Geraden mit verschiedener Steigung, was auf der jeweils unterschiedlichen Einstellung des Wertes $\mathrm{X}=m / e \pm \Delta m / e$ beruht.

Das Verfahren soll noch zur quantitativen Bestimmung anderer Substanzen, wie z. B. verschiedener Toxine, angewendet werden.

\section{W. D. Koller}

Bundesforschungsanstalt $f$. Lebensmittelfrischhaltung Institut $f$. Chemie u. Technologie

D-7500 Karlsruhe 1

Engesserstr. 20

Deutschland

$1.1 \mathrm{~m} / \mathrm{e}=$ Energieinhomogenität der Ionen mit der Masse $X=m / e$.

\section{Über einen spezifischen dünnschicht- chromatographischen Nachweis von Morphin}

On a Specific Detection of Morphine

by Thin-Layer Chromatography

ERnst KLUG

Institut für gerichtliche und soziale Medizin der Freien Universität Berlin

Eingegangen am 17. November 1971

Zum Nachweis von Morphin in Drogen, Tabletten und Zubereitungen der verschiedensten Art stehen seit seiner Entdeckung durch Sertürner $(1805 ; 1817)$ zahlreiche Analysenverfahren zur Verfügung. Ursprünglich wurden vor allem Kristall- und Farbreaktionen benutzt, deren sichere Anwendung jedoch weitgehend vorgereinigte Verbindungen voraussetzte. Einen entscheidenden Fortschritt bedeutete die Einführung der verschiedenen chromatographischen Arbeitsweisen, die heute in der Regel zur 